\title{
Inhibición del Trabajo de Parto Prematuro con Nifedipina
}

\author{
Dres.: Jairo de la Cruz Segura*, Ana Luiza Da Crus Rios de Sánchez**, \\ y Néstor Meneses** \\ HOSPITAL MILITAR CENTRAL \\ FACULTAD DE MEDICINA NUEVA GRANADA \\ SECCION GINECOLOGIA Y OBSTETRICIA
}

El parto pretérmino es un factor de suma importancia en la morbilidad y mortalidad perinatal $(1,2$, 3 ). A pesar de todo el conocimiento acumulado sobre la fisiología uterina y fisiopatología del trabajo de parto prematuro, las causas subyacentes son todavía desconocidas $(1,2,4)$.

Diversas drogas y esquemas terapéuticos han sido utilizados con diferentes tasas de éxito y efectos adversos en la madre y/o recién nacido $(1,3,4,5$, $6,7,8,9)$.

Desde hace varios años, se han utilizado los antagonistas del calcio como medicación antianginosa por su mecanismo de disminuir la entrada del calcio al medio intracelular y, consecuentemente, la actividad miocárdica. Recientemente han aparecido en la literatura informes sobre la eficacia de los calcio antagonistas en disminuir o impedir la contracción del músculo uterino, $(10,11,12,13)$.

El presente trabajo tiene por objeto presentar nuestra experiencia en 12 pacientes a las cuales se administró NIFEDIPINA (ester dimetílico del ácido $4-(2$, nitrofenil) $-2-6$ dimetil-1, 4 dihidro piridina $-3,5$ carboxílico) como agente uteroinhibidor.

\footnotetext{
* Profesor Asociado

** Residentes
}

La NIFEDIPINA es absorbida rápida y completamente después de la administración vía oral; su concentración máxima se alcanza entre los 15 y 90 minutos y la vida media es de 2 a 3 horas siendo excretada en las heces y orina $(70 \%)(1)$. Esta droga previene la entrada de calcio, al parecer, a través de los canales voltaje dependientes. Se sugiere que eso se produce por un efecto alostérico más que por una competición directa con el calcio para la entrada a través de esos canales (4). Los efectos colaterales de los calcioantagonistas considerados genéricamente, abarcan la vasodilatación que puede llevar a hipotensión, taquicardia refleja y efecto cardiovascular directo, específicamente, lentificando la conducción atrioventricular (4).

\section{MATERIAL Y METODOS}

Se estudiaron prospectivamente las pacientes que ingresaron al Hospital Militar Central entre junio de 1986 y junio de 1987 que llenasen los estrictos criterios seleccionados para el diagnóstico de Amenaza de Parto Pretérmino (APP) y previa autorización para la administración del fármaco.

El diagnóstico se basó en la determinación lo más precisa de la edad gestacional, actividad uterina y determinación de patología asociada a la APP.

Edad Gestacional: Se determinó por fecha de última menstruación, altura uterina y ecografía. 
Actividad Uterina: Se detectó clínicamente mediante la palpación de las contracciones uterinas, las cuales fueron de una frecuencia mayor o igual a 8 por hora o mayores o iguales a 4 en 20 minutos y documentadas con trazado de monitoría. Además la evaluación cervical con borramiento del $80 \%$ o dilatación de $2 \mathrm{~cm}$ o cambio cervical documentado.

A toda paciente se solicitó parcial de orina y cuadro hemático.

Pacientes con antecedentes o presencia de enfermedad cardiovascular, trastornos hipertensivos del embarazo, ruptura prematura de membranas, sospecha de retardo del crecimiento intrauterino, dilatación mayor de $4 \mathrm{~cm}$ y borramiento mayor del $50 \%$ no fueron incluidas en la presente investigación.

A todas las pacientes del presente estudio se les monitorizó la frecuencia cardíaca fetal y la actividad uterina y estuvieron constantemente vigiladas en pantalla. La monitorización hemodinámica materna se efectuó mediante el control de la frecuencia cardíaca y tensión arterial por personal médico, durante las primeras 6 horas.

La dosis inicial de la NIFEDIPINA fue de $30 \mathrm{mg}$ : $10 \mathrm{mg}$ sublinguales y $20 \mathrm{mg}$ vía oral. Posteriormente se administraron $10 \mathrm{mg}$ vía oral o $10 \mathrm{mg}$ vía oral y 10 sublingual a intervalos de 2 a 6 horas según la respuesta de la paciente en términos de intensidad y duración de las contracciones uterinas, cambios cervicales y considerando el monitoreo hemodinámico materno fetal. Si la respuesta fue rápida (entre los primeros 60 minutos) y duradera (ausencia completa de contracciones en las 6 horas siguientes) se administró solamente la dosis inicial. A las demás pacientes se fue reduciendo la dosis $(10 \mathrm{mg}$ cada 6 horas, después cada 8 horas y suspensión definitiva en el curso de 3 a 4 días).

A las pacientes en que no se obtuvo disminución de la dinámica uterina o hubo progreso en el trabajo de parto se inició manejo con Terbutalina: 10 mg disuelto en $500 \mathrm{cc}$ de $\mathrm{DAD}$ al $5 \%$ a razón de 10 a 15 microgramos por minuto endovenosos. A toda paciente entre la semana 28 y 34 se le efectuó maduración pulmonar con corticoides (Dexametasona $12 \mathrm{mg}$ IM c/ 12 h solamente 2 dosis).

\section{RESULTADOS}

Se administró NIFEDIPINA a 12 pacientes $\mathrm{cu}$ yos datos presentamos en el cuadro 1 :

\section{CUADRO 1}

\begin{tabular}{|cccccllrl|}
\hline No. Pac. E.G. & $\begin{array}{c}\text { Actividad } \\
\text { Uterina }\end{array}$ & $\begin{array}{r}\text { Dosis Total } \\
\text { (mg) }\end{array}$ & Monitor & $\begin{array}{l}\text { Utero } \\
\text { Inhibición }\end{array}$ & Dur (h) RN \\
\hline 1 & 30 & $4 / 20$ & B & 50 & React. & Positiva & 6 & AEG \\
2 & 35 & $3 / 10$ & R & 370 & React. & Positiva & 96 & AEG \\
3 & 34 & $2 / 10$ & B & 180 & React. & Positiva & 96 & AEG \\
$4^{*}$ & 23,5 & $2 / 10$ & L & 110 & React. & Positiva & 24 & AEG \\
5 & 31,3 & $4 / 10$ & B & 30 & React. & Positiva & 6 & AEG \\
6 & 34 & $2 / 10$ & B & 30 & React. & Positiva & 6 & AEG \\
$7^{*}$ & 32 & $3 / 10$ & B & 70 & React. & Positiva & 24 & AEG \\
8 & 30 & $4 / 10$ & B & 90 & React. & Positiva & 24 & AEG \\
$9^{*}$ & 30 & $2 / 10$ & B & 30 & React. & Negativa & 2 & AEG \\
10 & 35,5 & $3 / 10$ & B & 40 & React. & Negativa & 4 & AEG \\
11 & 36 & $4 / 20$ & B & 40 & React. & Negativa & 4 & AEG \\
12 & 26 & $3 / 10$ & B & 40 & React. & Negativa & 2 & AEG \\
\hline
\end{tabular}

$*$ =Infección Urinaria; $\mathrm{EG}=\mathrm{Edad}$ Gestacional $; \mathrm{AEG}=$ Recién nacido adecuado a la edad gestacional; $B=$ contracción uterina de buena intensidad; $\mathrm{R}=$ contracción uterina de regular intensidad; React. $=$ monitoría fetal externa reactiva.

La edad media de las pacientes fue de 26,4 años con edades comprendidas entre los 20 y los 37 años. La paridad osciló entre 0 a 4 hijos con un promedio de 2,5 hijos.

El incremento de la frecuencia cardíaca en pacientes manejadas con NIFEDIPINA fue en promedio de 8 latidos por minuto con rangos entre 0 y 24 latidos. La variación en las cifras tensionales sistólicas se manifestó en una disminución promedio de $10.5 \mathrm{~mm}$ de $\mathrm{Hg}(0$ a $30 \mathrm{~mm} \mathrm{Hg})$ y en la diastólica de $7,5 \mathrm{~mm} \mathrm{Hg}(0$ a $20 \mathrm{~mm} \mathrm{Hg})$. Este cambio no es estadísticamente significativo; ni causó cambios en la frecuencia cardíaca fetal que fueran detectables en la monitoría. Tampoco hubo necesidad de suspender la administración de la droga por repercusión cardíaca u hemodinámica en la madre. Es importante anotar que ninguna paciente presentó valores sistólicos menores de $100 \mathrm{~mm} \mathrm{Hg} \mathrm{ni} \mathrm{diastó-}$ licos menores de $60 \mathrm{~mm} \mathrm{Hg}$.

Cuatro de las pacientes $(33,3 \%)$ presentaron cefalea, tipo pulsátil, bitemporal que cedió con la utilización de compresas frías locales sin requerir terapia adicional. Como cosa curiosa, después de la administración de NIFEDIPINA, aun cuando persistieron las contracciones uterinas, las pacientes referían una mejoría subjetiva del dolor. Las pacientes toleraron bien el medicamento.

Una de las pacientes (No. 8), cursaba con embarazo gemelar y no hubo necesidad de cambiar la dosis o el manejo de esa paciente; posteriormente entró en trabajo de parto, a las 37 semanas y fue sometida a cesárea, por prímer gemelo en pelvis, sin complicaciones. 
Se obtuvieron 13 RN, 7 de sexo femenino y 6 de sexo masculino. El peso osciló entre 2480 y 3200 g. Los recién nacidos fueron valorados por el servicio de Pediatría del Hospital Militar Central y la calificación de Apgar osciló entre 7 y 9 sobre 10 al minuto. Todos los recién nacidos han evolucionado satisfactoriamente, hasta el momento. En ninguno se detectó alteración morfológica, metabólica o neurológica al momento del nacimiento o en controles posteriores.

En el grupo de las pacientes que no respondieron a la NIFEDIPINA una (No. 9) tampoco respondió a la Terbutalina por lo que se administraron ambas drogas conjuntamente $(10$ microgramos/minuto de terbutalina $+10 \mathrm{mg}$ v.o. de Nifedipina c/6 horas) obteniéndose buena respuesta sin incremento de los efectos colaterales de ninguna de las dos. Se obtuvo posteriormente un $\mathrm{RN}$ a las 40 semanas de embarazo de 3.200 g con Apgar de 9/10 y 10/10.

\section{DISCUSION}

Inicialmente se planeó utilizar las dosis descritas en los estudios de Ulmested; o sea, $30 \mathrm{mg}$ iniciales seguidos por $10 \mathrm{mg}$. vía oral cada tres horas por tres días (3). El objetivo de éste trabajo era retardar el parto mientras se inducía la maduración pulmonar con corticoides. Este autor informa uteroinhibición satisfactoria $(72 \mathrm{~h})$ en 10 pacientes con tal esquema.
En nuestra experiencia, la dosis anterior fue insuficiente para manejar la amenaza de parto pretérmino. El intervalo de administración de la droga necesario para mantener la uteroinhibición fue reducido a 2 horas, lo que es fácilmente comprensible teniendo en cuenta la vida media de la misma. Esto conllevó a un incremento importante de la dosis total administrada, hasta $370 \mathrm{mg}$ en 4 días, requeridos para la paciente número 2.

Estudios de Ulmstem, Csapo y posteriormente Forman y colaboradores, con calcio antagonistas "in vitro" e "in vivo" demuestran que la abolición de la actividad eléctrica del músculo uterino es dependiente de la concentración de la droga. En nuestro trabajo pudimos comprobar la acción limitada al tiempo de la administración y el gran margen de seguridad ofrecido por la Nifedipina. Creemos que la ausencia de efectos colaterales a nivel cardíaco, tanto materno como fetal, se debió al acúmulo del fármaco en el útero hipertrofiado del embarazo.

Pensamos que son necesarios estudios complementarios con un mayor número de pacientes para poder establecer un esquema terapéutico realmente eficaz y seguro. La uteroinhibición lograda en 8 de las 12 pacientes estudiadas y el amplio margen de seguridad ofrecido por la droga, cuando fue necesario utilizar dosis mucho más altas que las descritas en la literatura, constituyen un estímulo para continuar la investigación de los calcio antagonistas como alternativa o adyuvante de la terapia en la amenaza de parto pretérmino.

\section{BIBLIOGRAFIA}

1. CARSON: Premature Labor; “in"' Current Problems in Obstetrics, 1985.

2. HUSZAR, G. et al.: Miometriun and uterine cervix in normal and Preterm labor; N. Eng. J. of. Med. Vol. 311 No. 9. (571-580). Ago. 1984.

3. ARIAS, F. et al.: "Preterm Labor" in High Risk Pregnancy and Delibery"; Mosby co. 1984.

4. ROBERTS, J.M. et al.: "Estado actual del conocimiento de los mecanismos farmacológicos en la prevención del parto pretérmino'; C.G.O. Vol. 3, 1984 (757-774).

5. CARITIS, S.N. et al.: “A double blind study comparing Ritodrine and Terbutaline in the treatment of Preterm labor'; Am. J. Obstet. Gynecol. Vol. 150 No.1. Sep. 1984 (7-14).

6. HAHN, D.W. et al.: "Evaluation of drugs for arrest of Premature Labor in a new animal model"; Am. J. Obst. Gynecol Vol. 148 No. 6.March, 1984 (775-778).

7. CORENBROT, C.C. et al.: "The cost effectiveness of stopping preterm labour with betaadrenergic treat- men'; New England. J. Med. Vol. 310 No.11. March $1984(691-696)$.

8. KEIRSE, M.J. et al.: “A survey of tocolytic drug treatment in Premature Labour'; British J. Obst. and Gynecol, mayo 1984 (424-430).

9. VALENZUELA, G. et al.: "Follow-up hidration and sedation in the pretherapy of premature labor'; Am J. Obst. Gynecol. Vol. 147. Oct. 1983 (396-398).

10. SCHWARCZ, R.; CAPURRO, H. et al.: Manejo perinatal de la prematurez; CLAP No. 755, 1978.

11. ULMSTEM, U. et al.: "Relaxing Effects on Nifedipine on the no pregnan human uteros in vivo and in vitro"; Obst. Gynecol. Vol. 52 Oct. 1978 (436 - 441).

12. CSAPO, A.L. et al.: "Deactivation of the uterus during normal and Premature Labor by the calcium antagonist Nicardipine"'; Am. J. Obst: Gyn. Vol. 142 No.5, March 1982.

13. FORMAN, A., ANDERSON, KE., WINGERUP, L.: Treatment of the premature labor with calcium antagonist Nifedipine. Arch Gynecol 229. 1. 1981. 